\title{
Dynamical scaling of oxygen ordering in YBa2Cu3O7-
}

Poulsen, Henning Friis; Andersen, Niels Hessel; Andersen, Jørgen Vitting; Bohr, Henrik; Mouritsen, Ole G.

Published in:

Physical Review Letters

Link to article, DOI:

10.1103/PhysRevLett.66.465

Publication date:

1991

Document Version

Publisher's PDF, also known as Version of record

Link back to DTU Orbit

Citation (APA):

Poulsen, H. F., Andersen, N. H., Andersen, J. V., Bohr, H., \& Mouritsen, O. G. (1991). Dynamical scaling of oxygen ordering in YBa2Cu3O7-. Physical Review Letters, 66(4), 465-468.

https://doi.org/10.1103/PhysRevLett.66.465

\section{General rights}

Copyright and moral rights for the publications made accessible in the public portal are retained by the authors and/or other copyright owners and it is a condition of accessing publications that users recognise and abide by the legal requirements associated with these rights.

- Users may download and print one copy of any publication from the public portal for the purpose of private study or research.

- You may not further distribute the material or use it for any profit-making activity or commercial gain

- You may freely distribute the URL identifying the publication in the public portal

If you believe that this document breaches copyright please contact us providing details, and we will remove access to the work immediately and investigate your claim. 


\title{
Dynamical Scaling of Oxygen Ordering in $\mathrm{YBa}_{2} \mathrm{Cu}_{3} \mathrm{O}_{7-\delta}$
}

\author{
Henning Friis Poulsen, ${ }^{(1),(2)}$ Niels Hessel Andersen, ${ }^{(1)}$ Jørgen Vitting Andersen, ${ }^{(2)}$ Henrik Bohr, ${ }^{(2)}$ and \\ Ole G. Mouritsen ${ }^{(2),(3)}$ \\ ${ }^{(1)}$ Physics Department, Risø National Laboratory, DK-4000 Roskilde, Denmark \\ ${ }^{(2)}$ Department of Structural Properties of Materials, The Technical University of Denmark, \\ Building 307, DK-2800 Lyngby, Denmark \\ ${ }^{(3)}$ Department of Physical Chemistry, The Technical University of Denmark, Building 307, DK-2800 Lyngby, Denmark
} (Received 13 November 1990)

\begin{abstract}
Computer simulation on a two-dimensional anisotropic lattice-gas model of oxygen ordering in high$T_{c}$ superconductors of the $\mathrm{YBa}_{2} \mathrm{Cu}_{3} \mathrm{O}_{7-\delta}$ type shows that the ordering dynamics obey algebraic growth laws which are different in the ortho-I and ortho-II phases. It is possible to relate this dynamical scaling behavior to a similar scaling in the experimentally observed temporal variation of the superconductivity transition temperature, hence suggesting a specific coupling between the coherence of oxygen order in the basal $\mathrm{Cu}-\mathrm{O}$ planes and the superconducting state.
\end{abstract}

PACS numbers: $64.60 . \mathrm{Cn}, 61.70 . \mathrm{Ng}, 68.35 . \mathrm{Fx}, 74.70 . \mathrm{Vy}$

Dynamics of nonequilibrium ordering processes in condensed matter is a general area which has proved of fundamental interest because of the substantial theoretical and experimental evidence which points towards a description of the dynamics in terms of temporal scaling invariance and universality. ${ }^{1-3}$ Nonequilibrium ordering processes are widespread in nature. Among the more well-known examples are phase separation and spinodal decomposition, order-disorder phenomena, and grain growth. Theoretically, ${ }^{1}$ the description of such phenomena is extremely difficult due to inherent far-fromequilibrium conditions. Experimentally, ${ }^{2}$ the major part of the information available derives from three-dimensional systems. However, progress with highresolution, time-resolved spectroscopy has recently provided dynamical data for low-dimensional systems, specifically concerning ordering processes in adsorbates on solid surfaces. ${ }^{4}$

The purpose of the present paper is to point to a class of systems, high- $T_{c}$ superconductors of the $\mathrm{YBa}_{2} \mathrm{Cu}_{3}-$ $\mathrm{O}_{7-\delta}$ type, as being interesting candidates for studying the dynamics of two-dimensional ordering process. First, we show by a specific model calculation of the dynamics pertaining to the oxygen ordering in the basal $\mathrm{Cu}-\mathrm{O}$ planes that this ordering process obeys dynamical scaling as characterized by an algebraic growth law

$$
\bar{R}(t) \sim t^{n}
$$

where $\bar{R}(t)$ is a time-dependent linear length scale of the ordered domains. The growth exponent $n$ is found to have a characteristic dependence on whether the ordering is of the ortho-I type $(n \simeq 0.50)$ or of the ortho-II type ( $n \simeq 0.25-0.35$ ). This dependence may be rationalized in terms of well-known phenomenological theories of domain growth, provided the nature of the domain walls are taken into account. Second, we show by a simple reanalysis of existing experimental data ${ }^{5}$ for the temporal variation of the superconducting transition temper- ature, $T_{c}(t)$, of $\mathrm{YBa}_{2} \mathrm{Cu}_{3} \mathrm{O}_{6.41}$ subsequent to a quench into the ortho-II phase that the equilibrium transition temperature, $T_{c}(T)=T_{c}(t \rightarrow \infty)$, is approached algebraically as

$$
\Delta T_{c}(t)=T_{c}(T)-T_{c}(t) \sim t^{-m},
$$

where $m \simeq 0.5 \simeq 2 n$. Hence, $\Delta T_{c}(t) \sim \bar{R}(t)^{-2}$; i.e., the transition temperature scales as the area of the coherent two-dimensional oxygen order in the ortho-II phase. Our theoretical predictions for the ordering dynamics at different compositions suggest that it would be of great interest to perform quenching experiments systematically as a function of oxygen content to further test the scaling property and to examine how this may be used as an indicator of the relation between the morphology of oxygen order and the superconductivity.

Our numerical simulations are based on the locally anisotropic two-dimensional lattice-gas model proposed by Wille, Berera, and de Fontaine ${ }^{6}$ to describe the oxygen occupancy and ordering on a square lattice in the basal $\mathrm{Cu}-\mathrm{O}$ planes of $\mathrm{YBa}_{2} \mathrm{Cu}_{3} \mathrm{O}_{7-\delta}$. This model has been used successfully to describe a variety of thermodynamic equilibrium and nonequilibrium data for this material. ${ }^{7}$ The effective Hamiltonian of the model is given by

$\mathscr{H}=-V_{1} \sum_{i, j}^{\mathrm{NN}} n_{i} n_{j}-V_{2} \sum_{i, j}^{\mathrm{NN}(\mathrm{Cu})} n_{i} n_{j}-V_{3} \sum_{i, j}^{\mathrm{NN}} n_{i} n_{j}$,

where $n_{i}=0,1$ is an occupation variable and $V_{1}$ is the nearest-neighbor (NN) interaction strength. Interactions between a pair of oxygen atoms at next-nearestneighbor (NNN) sites are given by $V_{2}$ or $V_{3}$ depending on whether or not the pair is bridged by a $\mathrm{Cu}$ atom. The oxygen concentration is given by $c=\left\langle n_{i}\right\rangle=(1-\delta) / 2$.

We are going to focus only on the physically relevant case, $\quad V_{2}>0>V_{3}>V_{1}$, and oxygen concentrations $c \leq 0.50$. This choice of parameters leads at low temperatures to a strong tendency towards creation of diagonal $\mathrm{CuO}$ chains and chains of vacant oxygen sites, in effect 


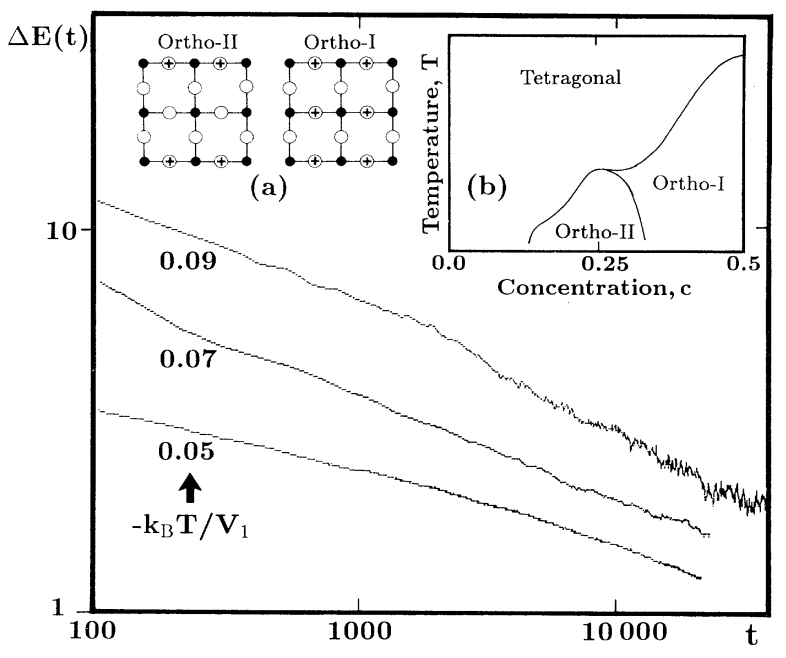

FIG. 1. Log-log plot of the excess energy $\Delta E(t)$ vs time (given in units of $\mathrm{MCS} / \mathrm{S}$ ) for quenches to different temperatures, $k_{B} T / V_{1}$, in the ortho-II phase. The oxygen concentration is fixed at $c=0.25$. Inset (a): The structure of the ground-state oxygen ordering in the ortho-II and ortho-I phases $(\bullet$, oxygen; $\oplus$, copper). Inset (b): The phase diagram of the model in Eq. (3).

making the structure and dynamics influenced by onedimensional effects. ${ }^{8}$ The phase diagram is shown schematically in inset (b) of Fig. 1. The diagram contains a high-temperature tetragonal phase and two lowtemperature orthorhombic phases, ortho-I and ortho-II. ${ }^{9}$ At $c=0.50$, the ground state is a simple twofolddegenerate structure consisting of alternating filled and vacant chains of oxygen sites. At $c=0.25$, a fourfolddegenerate structure is stable with three vacant chains alternating with one filled chain. The oxygen ordering of the orthorhombic phases is illustrated in Fig. 1. At nonstoichiometric concentrations phase separation does not occur. Rather, chain-diluted versions of the ortho-I structure of chain-diluted versions of the ortho-II structure are prevalent. Fitting the interaction parameters, via an equilibrium Monte Carlo calculation, to the recently reported neutron powder diffraction data of Andersen, Lebech, and Poulsen ${ }^{10}$ we have obtained the preliminary values $-V_{1} / k_{B}=4300 \mathrm{~K}$ and $\left(V_{2} / V_{1}, V_{3} / V_{1}\right)$ $=(-0.36,0.12)$. This set of values, which will be used throughout the present paper, leads to transition temperatures to the tetragonal phase around $-0.55 V_{1} / k_{B}$ and $-0.13 V_{1} / k_{B}$ for $c=0.50$ and $c=0.25$, respectively.

We have performed Monte Carlo simulations of quenches from infinite temperature to different temperatures well inside the ortho-I and ortho-II phases for different fixed oxygen concentrations. The dynamics is described by a density-conserving Kawasaki algorithm involving isotropic NN and NNN vacancy-particle exchange. The time $t$ is measured in Monte Carlo steps

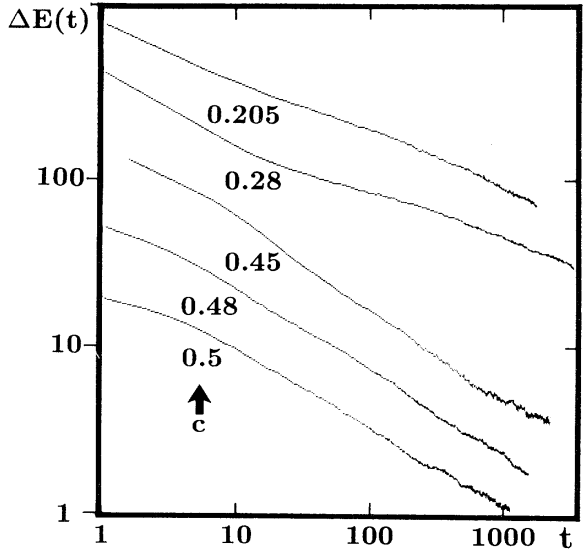

FIG. 2. Log-log plot of the excess energy $\Delta E(t)$ vs time (in units of MCS/S) for quenches into the ortho-II and ortho-I phases at different oxygen concentrations $c$ as indicated in the figure.

per site (MCS/S). The simulations are carried out on a variety of different lattices sizes, $L \times L, L=100,180$, 260 , and 400 , in order to eliminate possible size effects. The results reported below are believed to be size independent. To monitor the rate of growth we have calculated the temporal evolution of the excess energy, $\Delta E(t)=E(t)-E(T)$, where $E(T)$ is the equilibrium energy. As a measure of the total length of the domainboundary network, $\Delta E(t)$ is expected to scale as an inverse linear length scale, ${ }^{11} \Delta E(t) \sim \bar{R}(t)^{-1}$. To check this relation we have in some cases also used a lengthscale measure, $\bar{R}(t)$, derived from the full domain-size distribution function.

The evolution of the excess energy as a function of time at $c=0.25$ is shown in Fig. 1 for quenching temperatures $-k_{B} T / V_{1}=0.09,0.07$, and 0.05 . For the temperatures 0.07 and 0.05 we find an algebraic growth behavior with growth exponents $n=0.27(3)$ and $0.28(3)$, respectively. The corresponding result for $\bar{R}(t)$ is $n=0.27$ (3) and $0.28(3)$. For the higher temperature of 0.09 we find a transient of $n=0.28$ (3) in the decade from $t=100$ to 1000 followed by a crossover to a latestage value of $n=0.37(4)$. To further elucidate the temperature dependence, several simulations with low quenching temperatures are performed. For temperatures below 0.04 we find fairly low exponent values which may be attributed to crossover effects, since for quenches to $T=0$ the system freezes in and gets trapped in a metastable domain state. At $c=0.50$ and for intermediate temperatures, asymptotic exponent values of around $n=0.47$ (3) are observed over several decades. As an example, the relaxation of excess energy for the temperature $-k_{B} T / V_{1}=0.27$ is shown in Fig. 2 (lower curve). The growth exponents found for both $c=0.25$ and 0.50 are robust to the specific values chosen for the 
interaction parameters $V_{2}$ and $V_{3}$.

To investigate the dependence on concentration of the growth dynamics we have performed simulations at concentrations $c=0.205,0.28,0.45,0.48$, and 0.5 . The corresponding relaxation of excess energy is shown in Fig. 2. Simulations for quenches into the ortho-II phase are performed at a constant temperature $-k_{B} T / V_{1}=0.07$, estimated to be close to room temperature, while quenches into the ortho-I phase are done at temperatures roughly corresponding to half of the pertinent orthorhombic-totetragonal transition temperature. In all cases the growth data conform to algebraic growth laws with asymptotic exponents of $n=0.28(3), 0.29(3), 0.51(2)$, $0.45(3)$, and $0.47(2)$ for the series of concentrations listed above. The results for quenches into the ortho-I phase are obtained using only NN particle hopping. Corresponding sets of data for both NN and NNN hopping all gave effective exponents of $n \simeq 1.2$ for times up to $t=1000 \mathrm{MCS} / \mathrm{S}$ except from the $c=0.50$ case, where $n=0.47(3)$. We attribute these anomalously high exponent values to transient coalescence effects. ${ }^{12} \mathrm{We}$ conclude that the ordering dynamics is little affected by concentration variations within $\Delta c=0.05$ of the stoichiometric values of $c=0.50$ and 0.25 .

The different algebraic growth laws and the associated exponents found for oxygen ordering dynamics in the ortho-I and ortho-II phases may be rationalized within a general theoretical context. Except for the well-known effect of freezing in at low temperatures caused by metastability and thermal activation, ${ }^{3,11}$ the coherent picture which emerges from the results in Figs. 1 and 2 is that the ordering dynamics in the ortho-I phase is described asymptotically by an exponent $n=0.50$ and in the ortho-II phase by an intermediate-time exponent $n=0.25$ with a tendency to a crossover to $n \simeq 0.35$ at late times. The dynamics in both phases is characterized by a nonconserved order parameter. Hence one would expect the Lifshitz-Allen-Cahn growth law with $n=\frac{1}{2}$ to apply. ${ }^{1,3}$ This is consistent with our findings in the ortho-I phase. In the ortho-II phase, however, the lower asymptotic exponent as well as the intermediate-time exponent are more in accordance with the theory for spinodal decomposition (conserved order parameter) ${ }^{1}$ or for phase separation involving phases of different densities. ${ }^{13}$ These latter phenomena are both governed by long-range diffusion processes and hence covered by a LifshitzSlyozov growth law ${ }^{1}$ with $n=\frac{1}{3}$. By inspection of the different types of domain walls which characterize the ortho-II phase we find that in the late-time regime a dominant component of the domain-wall network consists of walls with excess or deficit local oxygen density. As first pointed out by Sadiq and Binder, ${ }^{11}$ the annealing of such a network requires transport of material over large distances. It may therefore be argued that the Lifshitz-Slyozov exponent should apply. This is indeed consistent with our results for the asymptotic exponent.
Furthermore, our finding of an intermediate-time exponent value of $n \simeq 0.25$ is also in agreement with the exponent values observed during the transient behavior in spinodal decomposition processes in a variety of model calculations. ${ }^{14}$ Finally, the effective temperature dependence of the growth exponent at $c=0.25$, specifically with regard to the low-temperature slowing down due to freezing in and the early crossover to Lifshitz-Slyozov behavior at high temperatures, is in close analogy with the results reported by Sadiq and Binder ${ }^{11}$ for fourfolddegenerate Ising models with $(2 \times 1)$ structures which also produce domain walls with excess or deficit density.

Turning now to a comparison of our theoretical prediction of dynamical scaling of oxygen ordering processes with pertinent experimental data we note that only very little data are available. In particular, no data exist for the dynamical structure factor of the oxygen ordering in $\mathrm{YBa}_{2} \mathrm{Cu}_{3} \mathrm{O}_{7-\delta}$. However, magnetic-susceptibility data are available ${ }^{5,15}$ which give the temporal evolution of the superconducting transition temperature, $T_{c}(t)$, for a quenched sample of $\mathrm{YBa}_{2} \mathrm{Cu}_{3} \mathrm{O}_{6.41}$, i.e., corresponding to a quench into the ortho-II phase. The data were analyzed by Jorgensen et al. ${ }^{5}$ in terms of different exponential functions of time. In light of the findings of the model calculation in the present paper we are led to reanalyze the experimental data in terms of an algebraic decay, Eq. (2), where the equilibrium transition temperature is determined by simple extrapolation. The results of this reanalysis are shown in Fig. 3 which demonstrates that a power-law fit with exponent $m \simeq 0.54$, Eq. (2), describes the entire set just as well as the ad hoc exponential fits proposed by Jorgensen et al. ${ }^{5}$ The experimental data in Fig. 3 should be compared with the upper curve in Fig. 2 corresponding to $c=0.205$. It is important to note that the exponent value pertinent to the experimental system in the ortho-II phase is that of the transient regime of a Lifshitz-Slyozov process due to the nature of the domain walls. This transient time regime becomes the relevant regime for the experimental system due to inherent limitations of the orthorhombic crystallinity by

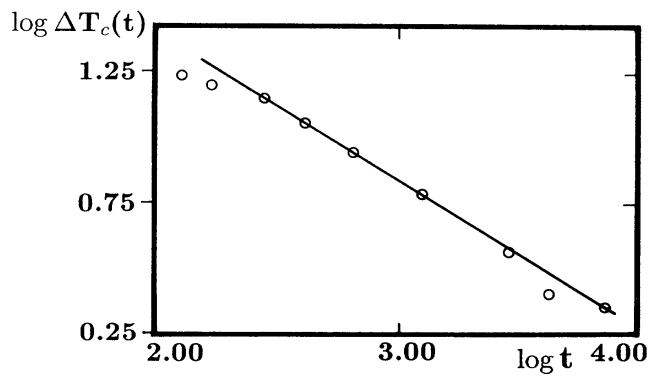

FIG. 3. $\log -\log$ plot of the relative variation, $\Delta T_{c}(t)$ in Eq. (2), of the superconducting transition temperature in $\mathrm{YBa}_{2}$ $\mathrm{Cu}_{3} \mathrm{O}_{6.41}$ as a function of time (in units of min) shown as obtained from an analysis of the experimental data (O) of Jorgensen et al. (Ref. 5). The solid line is the best power-law fit, $\Delta T_{c}(t) \sim t^{-0.54}$ 
twin domains which are only coherent over approximately 100 lattice spacings. ${ }^{16}$ Hence we conclude that the superconducting transition temperature varies algebraically and displays dynamical scaling with an exponent which is the same as that characteristic of the growth of the area of the coherent two-dimensional oxygen order in the ortho-II phase. The existence of a relation between $T_{c}$ and the oxygen ordering in the basal plane is further supported by a phenomenological theory of charge transfer. This theory, which is based on a counting of ordered oxygen clusters in the basal plane, is capable of reproducing the essential behavior of the experimentally observed variation of the equilibrium superconducting transition temperature with oxygen content. ${ }^{17}$ All this evidence strongly suggests that the properties of the superconducting state is coupled to the oxygen order in the $\mathrm{Cu}-\mathrm{O}$ planes of this material.

We have in this paper, based on a model calculation of the dynamics of oxygen ordering in the basal $\mathrm{Cu}-\mathrm{O}$ planes of high- $T_{c}$ superconductors of the $\mathrm{YBa}_{2} \mathrm{Cu}_{3} \mathrm{O}_{7-\delta}$ type, shown that this dynamics is characterized by algebraic growth laws with exponent values which depend on whether the evolving order is of the ortho-I or ortho-II type. We have pointed out that these ceramic superconductors therefore, by virtue of their extreme anisotropy with respect to oxygen diffusion, ${ }^{18}$ are unusually interesting candidates for studying aspects of dynamical scaling in low-dimensional systems. Furthermore, we have shown that the available experimental data on the time evolution of the superconducting transition temperature in the ortho-II phase also display dynamical scaling characterized by an exponent value which suggests that the transition temperature scales as the growth of the area of the coherent two-dimensional oxygen order in the basal $\mathrm{Cu}-\mathrm{O}$ planes of the material.

The results of the present paper are based on the basic physical principles of scaling symmetries in nonequilibrium ordering processes. The finding of the same scaling symmetry in two different material properties, oxygen order and superconducting transition temperature, is therefore unlikely to be accidental. Our results hence suggest that there is a specific coupling between the coherent oxygen order and the superconducting state of ceramic superconductors. Further systematic experimental time-resolved studies should be performed to test these ideas.

This work was supported by the Danish Natural Science Research Council under Grant No. J.nr. 11-7785 and by the Danish Technical Research Council under Grants No. J.nr. 16-4296.K and No. 16-4750.K. An illuminating discussion with Kurt Binder is greatly appreciated.
1J. D. Gunton, M. San Miguel, and P. S. Sahni, in Phase Transitions and Critical Phenomena, edited by C. Domb and J. L. Lebowitz (Academic, New York, 1983), Vol. 8, p. 267.

${ }^{2}$ For a recent review of the field of ordering dynamics, see Dynamics of Ordering Processes in Condensed Matter, edited by S. Komura and H. Furukawa (Plenum, New York, 1988).

${ }^{3}$ O. G. Mouritsen, in Kinetics of Ordering and Growth at Surfaces, edited by M. G. Lagally (Plenum, New York, 1990), p. 1.

${ }^{4}$ M. C. Tringides, Phys. Rev. Lett. 65, 1372 (1990); J.-K. Zuo, G.-C. Wang, and T. M. Lu, Phys. Rev. Lett. 60, 1053 (1988); P. K. Wu, M. C. Tringides, and M. G. Lagally, Phys. Rev. Lett. B 39, 7595 (1989).

${ }^{5}$ J. D. Jorgensen, S. Pei, P. Leightfoot, H. Shi, A. P. Paulikas, and B. W. Veal, Physica (Amsterdam) 167C, 571 (1990).

${ }^{6}$ L. T. Wille, A. Berera, and D. de Fontaine, Phys. Rev. Lett. 60, 1065 (1988).

${ }^{7}$ A. Berera and D. de Fontaine, Phys. Rev. B 39, 6727 (1989); N. C. Bartelt, T. L. Einstein, and L. T. Wille, Phys. Rev. B 40, 10759 (1989); Z.-X. Cai and S. D. Mahanti, Phys. Rev. B 40, 6558 (1989); C. P. Burmeister and L. T. Wille, Phys. Rev. B 40, 8795 (1989); T. Aukrust, M. A. Novotny, P. A. Rikvold, and D. P. Landau, Phys. Rev. B 41, 8772 (1990); J. V. Andersen, H. Bohr, and O. G. Mouritsen, Phys. Rev. B 41, 283 (1990).

${ }^{8}$ P. A. Rikvold, M. A. Novotny, and T. Aukrust (to be published).

${ }^{9} \mathrm{~A}$ third orthorhombic phase, the ortho-I phase [G. Ceder, M. Asta, W. C. Carter, M. Kraitchman, D. de Fontaine, M. E. Mann, and M. Sluiter, Phys. Rev. B 41, 8777 (1990)] will not be considered here. This phase, which has broken rotational symmetry, only consists of parallel $\mathrm{CuO}$ chains randomly translated with respect to each other.

${ }^{10}$ N. H. Andersen, B. Lebech, and H. F. Poulsen, Physica (Amsterdam) 172C, 31 (1990).

${ }^{11}$ A. Sadiq and K. Binder, J. Stat. Phys. 35, 517 (1984).

${ }^{12} \mathrm{We}$ have observed similar transient effects in the simple dilute lattice-gas model with purely repulsive interactions $\left(V_{2}=V_{3}=0\right)$.

${ }^{13}$ W. W. Mullins and J. Viñals, Acta Metall. 37, 991 (1989); O. G. Mouritsen, P. J. Shah, and J. V. Andersen, Phys. Rev. B 42, 4506 (1990).

${ }^{14}$ C. Roland and M. Grant, Phys. Rev. B 39, 11971 (1989); K. Yaldram and K. Binder, Acta Metall. (to be published); D. Huse, Phys. Rev. B 34, 7845 (1986); J. G. Amar, F. E. Sullivan, and R. D. Mountain, Phys. Rev. B 37, 196 (1988); G. F. Mazenko, O. T. Valls, and M. Zannetti, Phys. Rev. B 38, 520 (1988).

${ }^{15}$ B. W. Veal, H. You, A. P. Paulikas, H. Shi, Y. Fang, and J. W. Dowey, Phys. Rev. B 42, 4770 (1990).

${ }^{16}$ H. F. Poulsen, N. H. Andersen, and B. Lebech, Physica C (to be published).

${ }^{17}$ H. F. Poulsen, N. H. Andersen, J. V. Andersen, H. Bohr, and O. G. Mouritsen (to be published).

${ }^{18}$ S. J. Rothman, J. L. Routbort, and J. E. Baker, Phys. Rev. B 40, 8852 (1989). 